\title{
Unusual heavy ion injection events observed by Freja
}

\author{
M. Yamauchi ${ }^{1}$, L. Eliasson ${ }^{1}$, R. Lundin ${ }^{1}$, and O. Norberg ${ }^{2}$ \\ ${ }^{1}$ Swedish Institute of Space Physics, Box 812, S-98128 Kiruna, Sweden \\ ${ }^{2}$ Swedish Space Corporation - Esrange, Box 802, SE-98128 Kiruna, Sweden
}

Received: 3 March 2004 - Revised: 15 November 2004 - Accepted: 19 November 2004 - Published: 28 February 2005

\begin{abstract}
Heavy ion injection events (registered in the $\mathrm{C}+/ \mathrm{N}+/ \mathrm{O}+$ mass channel) into the ionosphere at $1700 \mathrm{~km}$ height are surveyed using the Swedish-German Freja satellite data. Heavy ion injections from the inner magnetosphere are mostly found in the nightside sector with a few exceptions that occur on the dayside. We report on these exceptional cases, particularly two specific events which seem to have a different generation/transport mechanism of heavy ions from the majority of cases: 1) mono-energetic heavy ion injection near local noon with oxygen first and protons later; and 2) a multiple heavy ion dispersion event at 9 MLT. Both events are unique without any similar events in terms of dispersion pattern during the more than 2 years of the entire Freja operation, and these events are found during the main phase of major magnetic storms (peak $D_{s t}$ is $-144 \mathrm{nT}$ and $-105 \mathrm{nT}$ ). The first event is probably of dayside origin but the exact mechanism to make both energy filter and mass filter is unknown. The second event can be traced back to one localized energization of dense oxygen with strong pitch-angle anisotropy within $1000 \mathrm{~km}$ distance from the spacecraft.
\end{abstract}

Key words. Ionosphere (Particle precipitation, Ion chemistry and composition) - Magnetosphere (Magnetospheric configuration and dynamics)

\section{Introduction}

Magnetospheric heavy ion injections into the ionosphere have rarely been discussed although they were already detected in the early 1970's (Shelley et al., 1972). One reason for this lack of reports may be related to the fact that no past low-altitude $(<3000 \mathrm{~km})$ satellite except for Freja (Lundin et al., 1994) and FAST (Carlson et al., 1998) had simultaneous mass and pitch angle resolution for the important energy range $0.1-10 \mathrm{keV}$ (typical for injecting plasma), so that one could not distinguish the composition of precipitating particles. Yet, some of the plasma injections into the ionosphere reported in the past (without mass spectrometers) are considered to contain substantial heavy ions. We pedagogically call them $\mathrm{O}+$ but they could also contain $\mathrm{N}+\mathrm{C}+$ or $\mathrm{Na}+$ (Chappell et al., 1982; Yau et al., 1993) because their

Correspondence to: M. Yamauchi

(m.yamauchi@irf.se) location and multiple energy characteristics suggest that the source is the opposite hemisphere or (oxygen-rich) ring current region (Quinn and McIlwain, 1979; Winningham et al., 1984; Bosqued et al., 1986; Mauk, 1986; Frahm et al., 1986; Hirahara et al., 1997). Since the escape of ionospheric ions is a common feature over the polar region in the form of ion beams or conics (e.g. Lundin et al., 1987; Norqvist et al., 1998; Moore et al., 1999), and since the inner magnetosphere is eventually filled with heavy ions during magnetic storms (Hamilton et al., 1988; Blanc et al., 1999), it is not surprising to observe heavy ion injections into the ionosphere from the inner magnetosphere.

Therefore, low-altitude identification and classification of heavy ion injections into the ionosphere are needed. Although Freja's altitude $(1700 \mathrm{~km})$ is not sufficiently low to extract the influx into the ionosphere from all downgoing ions, and although Freja's inclination $\left(63^{\circ}\right)$ is not high enough to cover the entire injection region, it provides a unique opportunity to extend our knowledge of global oxygen (or heavy ion) circulation.

Freja's three-dimensional ion composition spectrometer (TICS) provides high spatial/temporal resolution (about $80 \mathrm{~m}$ or $10 \mathrm{~ms}$ per energy step, and $3 \mathrm{~km}$ or $0.4 \mathrm{~s}$ per energy sweep) data for heavy ions and protons (Eliasson et al., 1994) with $30 \%$ energy resolution in its normal mode. Although it sometimes suffers from contamination from protons (this is inevitable for almost all mass-resolving ion spectrometers when the flux is high), it is capable of identifying primarily injecting species between proton and heavy ions, as shown in Fig. 1 (see the next section for details). Using this capability, various types of non-cusp proton injections observed by Freja are summarized by Hultqvist (2002) and the cusp injections by Norberg et al. (1994). However, the heavy ion injections observed by Freja have not been reported in the past.

We first briefly show an example and local time distribution of the heavy ion injection events. We then show two unique events which are found at unusual locations (both in dayside) with quite different characteristics from the majority of injections (i.e. nightside events). The first event is intense simultaneous ion injections in the post-noon ring current region with $\mathrm{O}+$ preceding $\mathrm{H}+$ at a narrow energy band, and the other event comprises multiple injections of heavy 
(a) Freja hot plasma data, orbit 3185, 1993-6-04

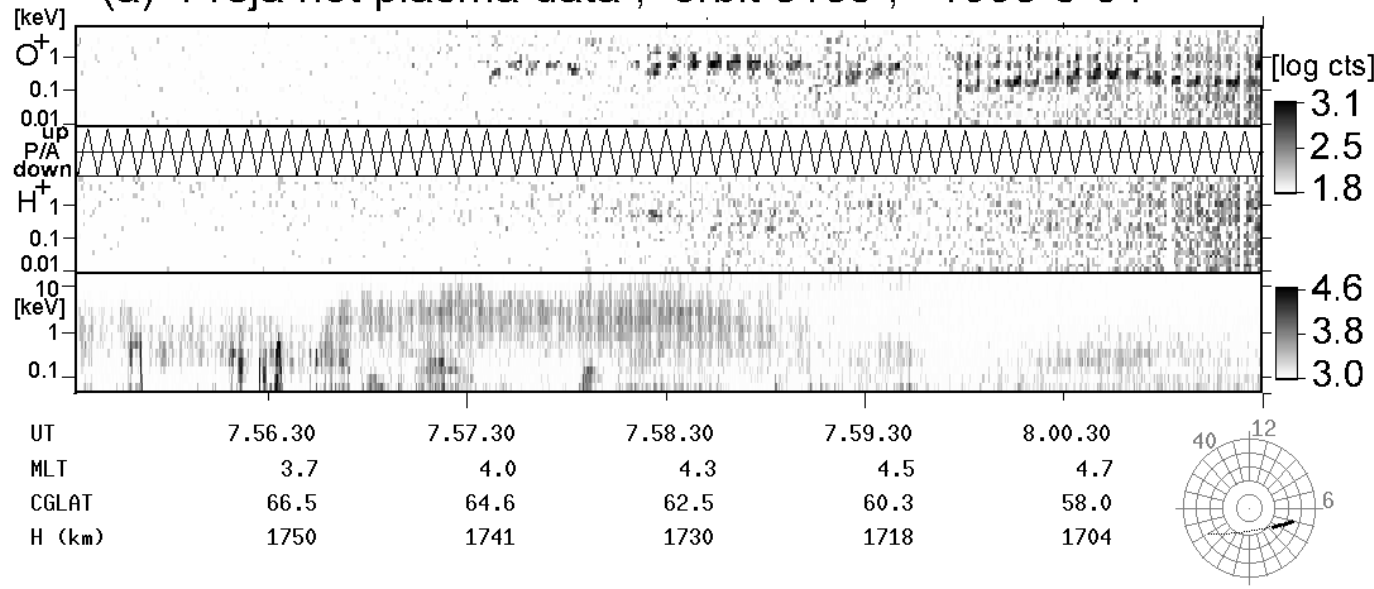

(b) Freja hot plasma data, orbit 1313, 1993-1-13

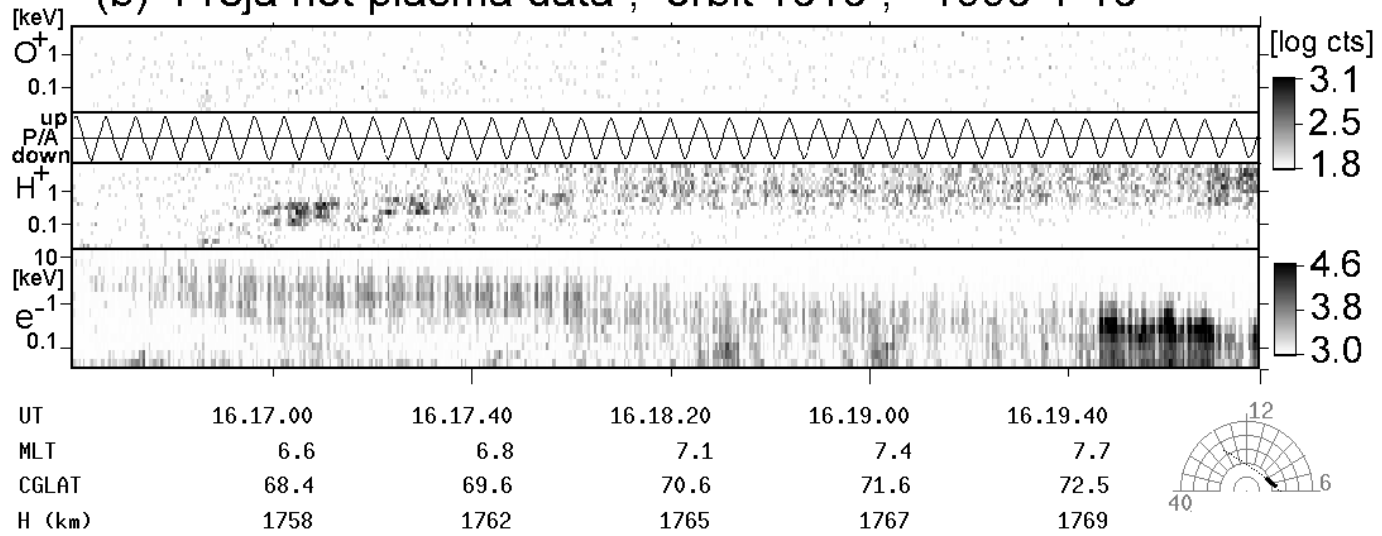

Fig. 1. Freja heavy ion $(0.01-10 \mathrm{keV})$, proton $(0.01-10 \mathrm{keV})$, and electron $(0.03-20 \mathrm{keV})$ spectrograms of commonly-found ion injections near or equatorward of the nightside auroral zone: (a) With heavy ions dominant (orbit 1652); (b) With protons dominant (orbit 1313). We chose a sector that covers the pitch angles (P/A) best for ions, and two sectors which are mostly parallel to the magnetic field (within 45 deg of the magnetic field) for electrons. CGLat=corrected geomagnetic latitude. Note that the legend "oxygen" (same for the other figures) means heavy ions and might contain $\mathrm{N}+$ and other heavy ions because the instrument cannot distinguish between them. We simply interpret them as oxygen of ionospheric origin.

ions, probably starting at the same time, from a single source very close to the satellite altitude. No similar phenomenon has been found during more than 2 years of Freja observation, nor has it been reported in the past.

\section{Ordinary heavy ion injection events}

Figure 1a shows an example of commonly-observed heavy ion injection events near the auroral zone. For reference we also show an example of commonly-observed proton injections in the auroral zone in Fig. 1b. Electron data (Boehm et al., 1994) is included to show the location relative to the region of thermal unstructured electrons at several $\mathrm{keV}$ range, which is traditionally called the central plasma sheet: CPS (Winningham et al., 1975; Woch and Lundin, 1993). While proton injections can be found in the entire auroral zone, heavy ion injections are found mostly inside or equatorward of the CPS. Heavy ion injections inside and around the auroral zone are mostly without consistent (or smooth monotonic) energy dispersion (i.e. the energy is either constant or fluctuating for more than $30 \mathrm{~s}$ ) in 30\% energy resolution data. For the case in Figure 1a, none of the injections at 07:57:35 UT (for $25 \mathrm{~s}$ ), 07:58:25 UT (for $35 \mathrm{~s}$ ), and 07:59:55 UT (for more than $90 \mathrm{~s}$ ) has monotonic dispersion, and hence they cannot be a simple time-of-flight signature (particles with high field-aligned velocity arrive first and particles with slow field-aligned velocity arrive later when they have the same source region). These injections must instead reflect the source structure and/or non-uniform convection (cf. Winningham et al., 1984).

The global distribution of the heavy ion injections from the Freja data (more than 6000 traversals, inclination $63^{\circ}$ ) is shown in Fig. 2. In this figure, however, we just simply show the distribution of "clear" or "strong" examples of heavy ion injections (types of Fig. 1a), and we exclude simultaneous proton and oxygen injections (e.g. cusp during magnetic 
storms, McFadden et al., 2003) to completely exclude the contamination effect, i.e. we selected events in which the peak of counts is found in the oxygen mass channel (proton is a minor component) for a given energy and time. Therefore, we may underestimate the number of oxygen injection events.

As is obvious from Fig. 2, the heavy ion injection events are found mainly on the nightside, whereas a very small portion of the heavy ion injection events is found on the dayside. Since almost all the oxygen injections are found inside or equatorward of the CPS even in traversals that cover the entire auroral oval and the polar cap boundary, the low inclination $\left(63^{\circ}\right)$ of the orbit does not affect this result very much, even though the chances of passing the dayside auroral oval are reduced. Meanwhile, the orbital coverage by Freja is uniform along MLT in the auroral zone. Therefore, the nightside preference is not an artifact of the orbit. The nightside events shown in Fig. 1 have characteristics consistent with the interpretations given by previous low-altitude studies (Winningham et al., 1984; Frahm et al., 1986).

\section{Unique events}

In Fig. 2 one can recognize that only six out of 198 clear injection events of heavy ions are found in the midday sector. Two of these six events (orbit \#6651 an orbit \#1103) have unique characteristics. We have never detected anything similar during more than 2 years of Freja observations at all MLT. The rest of the paper is dedicated to reporting these events.

\subsection{Midday heavy ion injection event}

The first exceptional example is shown in Fig. 3. It took place on 21 February 1994 (orbit \#6651) when the interplanetary and magnetospheric activity was extremely high with strong dawnward IMF (Yamauchi et al., 1996b). The geomagnetic condition during and previous to this traversal is given in Fig. 4a.

Nearly mono-energetic heavy ion injection $(0.3-1 \mathrm{keV}$ range) is observed at 13 MLT (13:31:30 UT). The cusp is significantly shifted dawnward during this event (seen until 13:27:30 UT) (Yamauchi et al., 1996b), and these heavy ions are eventually found in the afternoon sector far from the cusp. Both the injection location and the dispersion are similar to those of a stagnant plasma injection (SPI) event (Yamauchi et al, 1993), but the event is different in composition from SPI which is mainly composed of protons. In fact, SPI is recognized at 13:28:25-13:29:20 UT in the proton channel.

The heavy ion injection is immediately followed by a proton injection at $10-30 \mathrm{eV}$, and the energy ratio between these two species at any given time is constant at about 15-20. Since the simultaneous appearance suggests that they have the same velocity at each moment, the heavy ion is most likely $\mathrm{O}+$ (mass ratio 16$)$ rather than $\mathrm{N}+$ (mass ratio 14) or $\mathrm{C}+$ (mass ratio 12), if the heavy ions and $\mathrm{H}+$ originate from
Freja observation of Heaw Ion Injection $(>100 \mathrm{eV})$ 1992.10 - 1994.10 (orbit \# 300-10000)

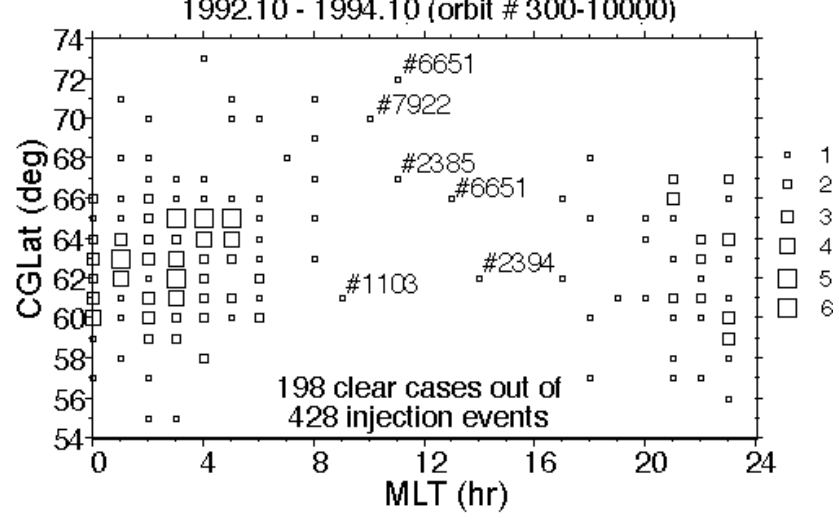

Fig. 2. Distribution of heavy ion injections observed by Freja. Each traversal with injection events (clear cases only) is registered in one $1^{\circ} \times 1 \mathrm{~h}$ bin, and the total number of traversals with injection events are plotted as squares (scaled by the size of the square). The number with \# is the orbit number.

the same region. This event took place during the storm time when $\mathrm{N}+$ was expected to be nearly as rich as $\mathrm{O}+$ in the magnetosphere (Yau et al., 1993); yet the energy band (width in $\%)$ of injecting ions is about the same for proton and heavy ions, indicating that one species $(\mathrm{O}+)$ dominated among $\mathrm{C}+$, $\mathrm{N}+$ and $\mathrm{O}+$.

With an energy of the downgoing ions of $\approx 300 \mathrm{eV}$ $(60 \mathrm{~km} / \mathrm{s}$ for $\mathrm{O}+)$ and the dispersion curve, i.e. the energy decrease from $0.32 \mathrm{keV}$ at $13: 31: 30$ to $0.18 \mathrm{keV}$ at 13:32:06, the time-of-flight distance is estimated to be $h \approx 1 \operatorname{Re}(=\Delta T * V 1 * V 2 / \Delta V$, where $V 1$ is the starting downward velocity, $V 2$ is the downward velocity after time duration of injection $\Delta T$, and $\Delta V=V 1-V 2$, e.g. Kletzing and Torbert, 1994). The velocity filter distance $\left(=h * V_{\text {satellite }} / V_{\text {convection }}\right)$ is then estimated to be at least $>10 \mathrm{Re}$. The latter means that the source must be either in the equatorial region or in the opposite hemisphere at around the same latitude (68 CGLat or $\mathrm{L}=7$ ). As a third alternative the source could be spatially dispersive. One cannot estimate the source distance in the last case.

The peculiarity of this event is that the heavy ions are registered first (at higher latitude) before protons are registered (at lower latitude). The time lag (or spatial separation) is seen even in the faint precursor at 13:31:10-13:31:30 UT in the oxygen channel and at 13:31:30-13:32:00 UT in the proton channel. Looking at the entire injection event, the average energy ratio between $\mathrm{O}+$ and $\mathrm{H}+$ is larger than the mass ratio (i.e. fast $\mathrm{O}+$ and slow $\mathrm{H}+$ ). The observed pattern (time/spatial lag with high energy ratio) is quite unusual. It is also puzzling from a modeling viewpoint because a simple finite (limited) temperature effect in the source region produces fast $\mathrm{H}+$ and slow $\mathrm{O}+$, i.e. the opposite pattern.

This unique observation raises two major questions: 1) what is the source location/mechanism and 2) what is the mass-filtering mechanism? If the source is bursty, we need to know the mechanism which creates oxygen first and protons 


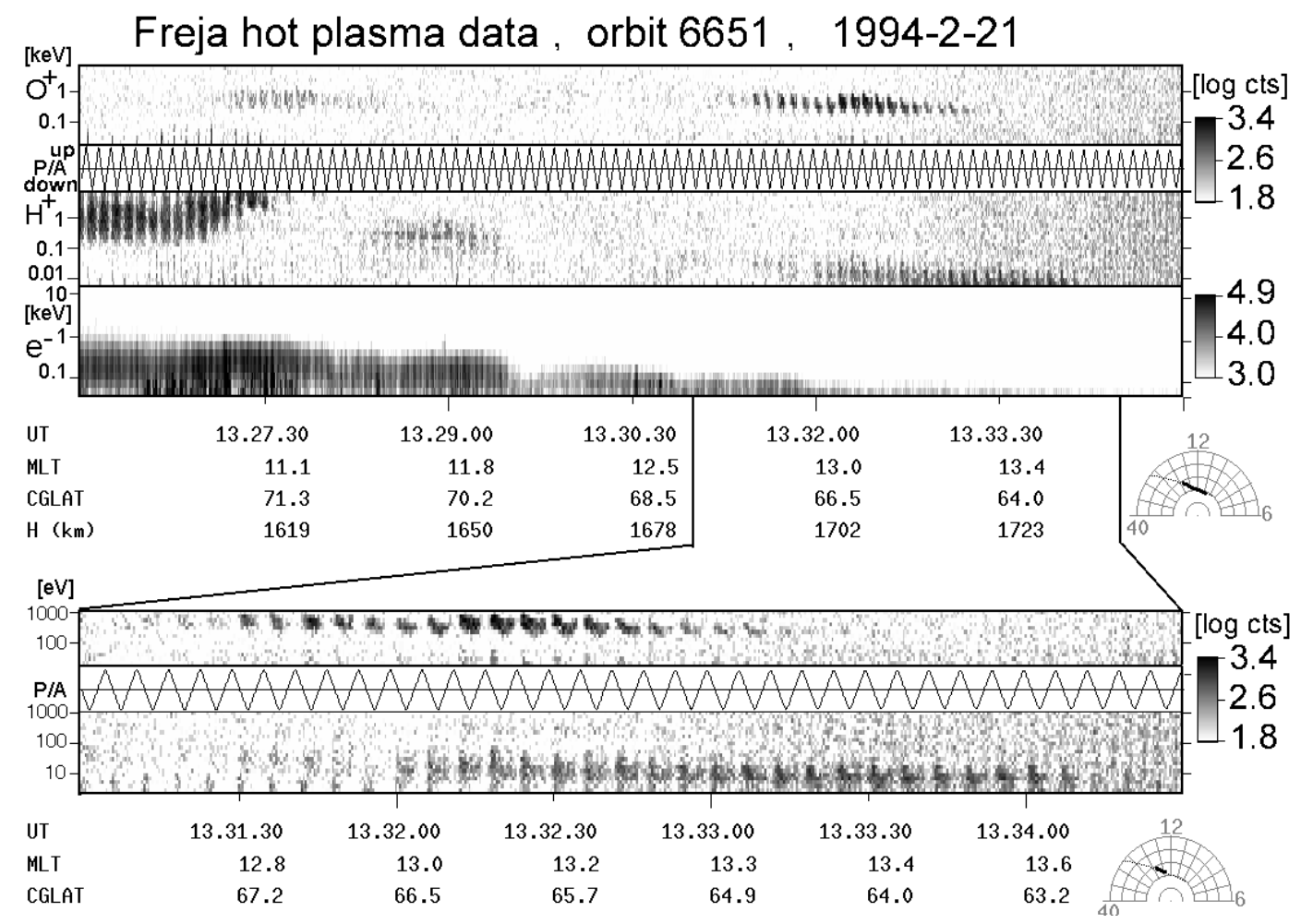

Fig. 3. Ion and electron spectrogram for Freja orbit 6651 (21 February 1994) with the same format as Fig. $1 . K_{p}$ is $7+$ and the solar wind is extremely disturbed (IMF is as strong as $50 \mathrm{nT}$ ).

later. If the source is steady, we need to know the mechanism that creates fast oxygen and slow protons.

To understand this unusual injection, we examine the general paths and sources of heavy ions, as illustrated in Fig. 5. The major sources of heavy ions known to date (Moore et al., 1999, and references therein) are the dayside ionosphere in the form of upgoing ions up to $\mathrm{keV}$ (marked (C) and (C2) in the figure), the nightside ionosphere in the form of ion beams or elevated conics over the auroral acceleration region up to tens $\mathrm{keV}$ (marked (B2) in the figure), and the nightside plasma sheet which is ultimately supplied from the ionosphere some time before (marked (A) and (B) in the figure). When the source is on the nightside, the ions drift either (A) eastward by the $\boldsymbol{E} \times \boldsymbol{B}$ drift or (B) westward by the gradient$B /$ curvature drift, making the entire dawnside or duskside inner magnetosphere rich in heavy ions. Such an oxygen-rich magnetosphere is in fact a characteristic of major magnetic storms (Blanc et al., 1999, and references therein). The dayside source is either (C) the Northern Hemisphere or (C2) the Southern Hemisphere.

The major magnetic storm condition during this period (see Fig. 4) supports the idea that the observed heavy ions might have come from the oxygen-rich ring current which has ultimately drifted from the nightside. However, there are some difficulties with this scenario.

The fate of the nightside source has been simulated by Ebihara et al. (2001), and the result predicts a wedge-like dispersion of trapped sub-keV particles. Although the simu- lation needs to be improved to include mass separation during the drift, the basic signature (e.g. dispersion curve and pitch-angle distribution) should be preserved according to the Cluster observation of sub-keV ring current ions (Yamauchi et al., 2005). This wedge-like signature is quite different from the observed energy-latitude dispersion. Furthermore, if the observed heavy ions come from the nightside (or oxygen-rich ring current), they must have been converted from a nearly trapped population to a nearly downgoing population only at 13 MLT and only during this magnetic storm. So far, to the authors' knowledge, no successful theory exists for such a process. The injection starting from $\mathrm{O}+$ instead of $\mathrm{H}+$ (this is the major peculiarity) is another difficulty because the drifting $\mathrm{O}+$ and $\mathrm{H}+$ are expected to have similar distributions (Ebihara, private communication, 2003) and hence we expect to see $\mathrm{H}+$ first and $\mathrm{O}+$ later, if the scattering mechanism works equally for both species. Therefore, we need an additional mass-filtering mechanism during the drift, if the source of this event is the nightside. The extremely high solar wind dynamic pressure and/or extremely large dawnward IMF during this period (Yamauchi et al., 1996b) might assist such an unusual mass-filtering and scattering, but this is no more than a speculation.

Therefore, we should consider explaining these oxygen ions by a dayside origin, i.e. either (C) or (C2) in Fig. 5, although we have no solid evidence to support the dayside source. It is worthwhile to point out that the observed location is in the vicinity of the conjugate region of the southern 

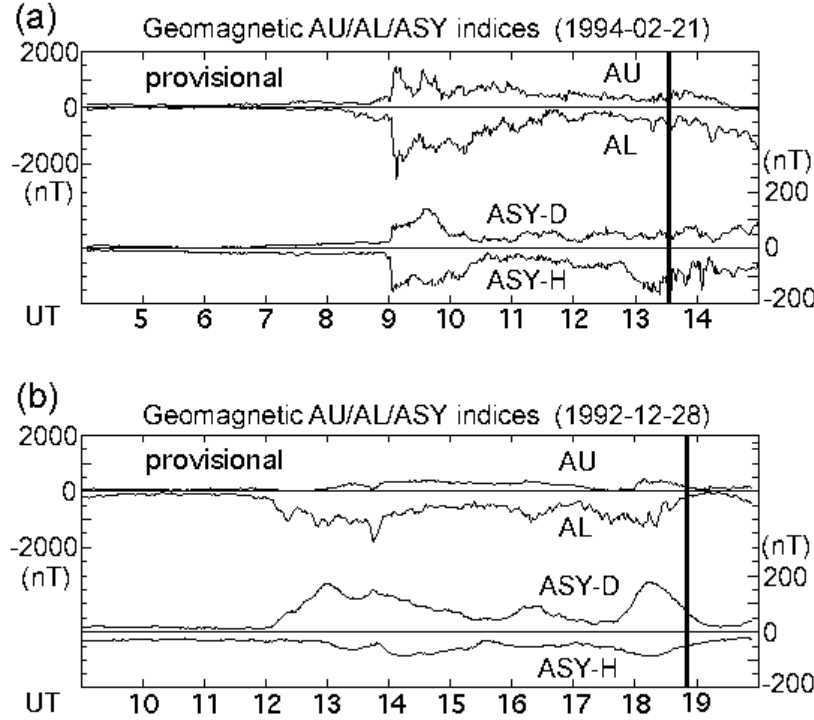

Fig. 4. AE and ASY indices (11-h plot) on (a) 21 February 1994 (cf. Fig. 3) and (b) 28 December 1992 (cf. Fig. 6). The thick vertical line indicates the Freja observation of the injection event. Both panels indicate major magnetic storms.

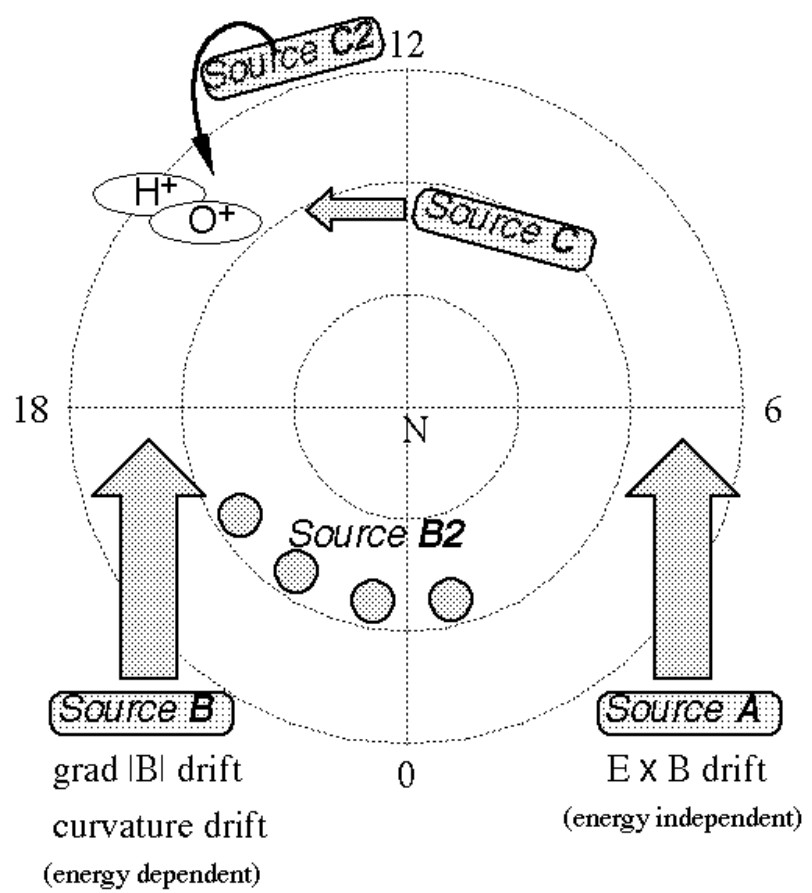

Fig. 5. Illustration of possible transport routes for the dayside heavy ions: (A) Eastward $\boldsymbol{E} \times \boldsymbol{B}$ drift from the nightside; (B) Westward gradient-B/curvature drift from nightside; (C) Dayside ionosphere.

cusp due to the unusually strong dawnward IMF. The dayside cusp, cleft, and its prenoon side are large source regions of ion escape from the ionosphere (Moore et al., 1999 and references therein).
However, the dayside origin also has some difficulty. If the observed heavy ions come from the opposite hemisphere, the responsible escape mechanism should give both $\mathrm{O}+$ and $\mathrm{H}+$ the same velocity with narrow energy ranges. This characteristic normally implies a velocity filter, but in this case we expect that the ions which are slower than a certain velocity are lost through the polar cap and only fast ions can stay within the closed magnetosphere. This is contrary to what is observed. Another problem is why Freja never detected the same type of events during its mission. To understand the unknown mechanism that generates this unique injection signature, we will have to wait until other spacecraft detect similar events.

\subsection{Heavy ion injection adjacent to the cusp}

In Fig. 3, one can also recognize another clear heavy ion injection at 13:27:20 UT, although it is fainter than the one discussed above (13:31:30 UT). Unlike the previous case, this injection is found adjacent to and equatorward of the cusp (which ends at 13:27:30 UT). Since O+ injection into the cusp is a common phenomenon during magnetic storms according to the FAST observations (McFadden et al., 2003), one might connect this injection to the same type as the FAST observations.

However it is not that simple. The heavy ion injection in the cusp is always somewhat fainter in the Freja data than in the FAST data. In other words, the downgoing heavy ion detection by Freja is either shorter in duration or lower in flux (or both) than that by FAST. As a consequence, the example in Fig. 3 is the only observation of clear heavy ion injection in the cusp with sufficient influx to separate from the proton contamination among more than 100 Freja cusp traversals (over more than two years).

More precisely, Freja detected a total of four "clear" cases of heavy ion injections relatively close to the cusp during its mission. However, three of them (orbits \#2385, \#2394, and \#7922; locations are shown in Fig. 2) are found inside the CPS, and are completely away from the cusp. The last one is the present observation. Hence, we do not know at present if it is related to the cusp at all.

Another problem in associating this heavy ion injection with the cusp O+ observed by FAST (McFadden et al., 2003) is that the oxygen energy is lower than the proton energy (both at 13:27:30 UT) contrary to the common cases observed by FAST. Therefore, we should also consider other explanations, for example: drifted ions from the nightside; escaping ionospheric ions reflected at the high-altitude cusp; or escaping ions from the opposite hemisphere. At present, we cannot identify the source mechanism and the source region for this specific case. The energy ratio of these heavy ions $(0.2-1 \mathrm{keV})$ and the SPI at $13: 28: 30 \mathrm{UT}(0.1-0.3 \mathrm{keV})$ is about 3 and is quite different from the energy ratio of 15-20 during the mono-energetic heavy ion injection (13:31:30 UT) discussed in the previous subsection.

It is rather puzzling that Freja did not detect the clear O+ injection that FAST detected during magnetic storms. Here 


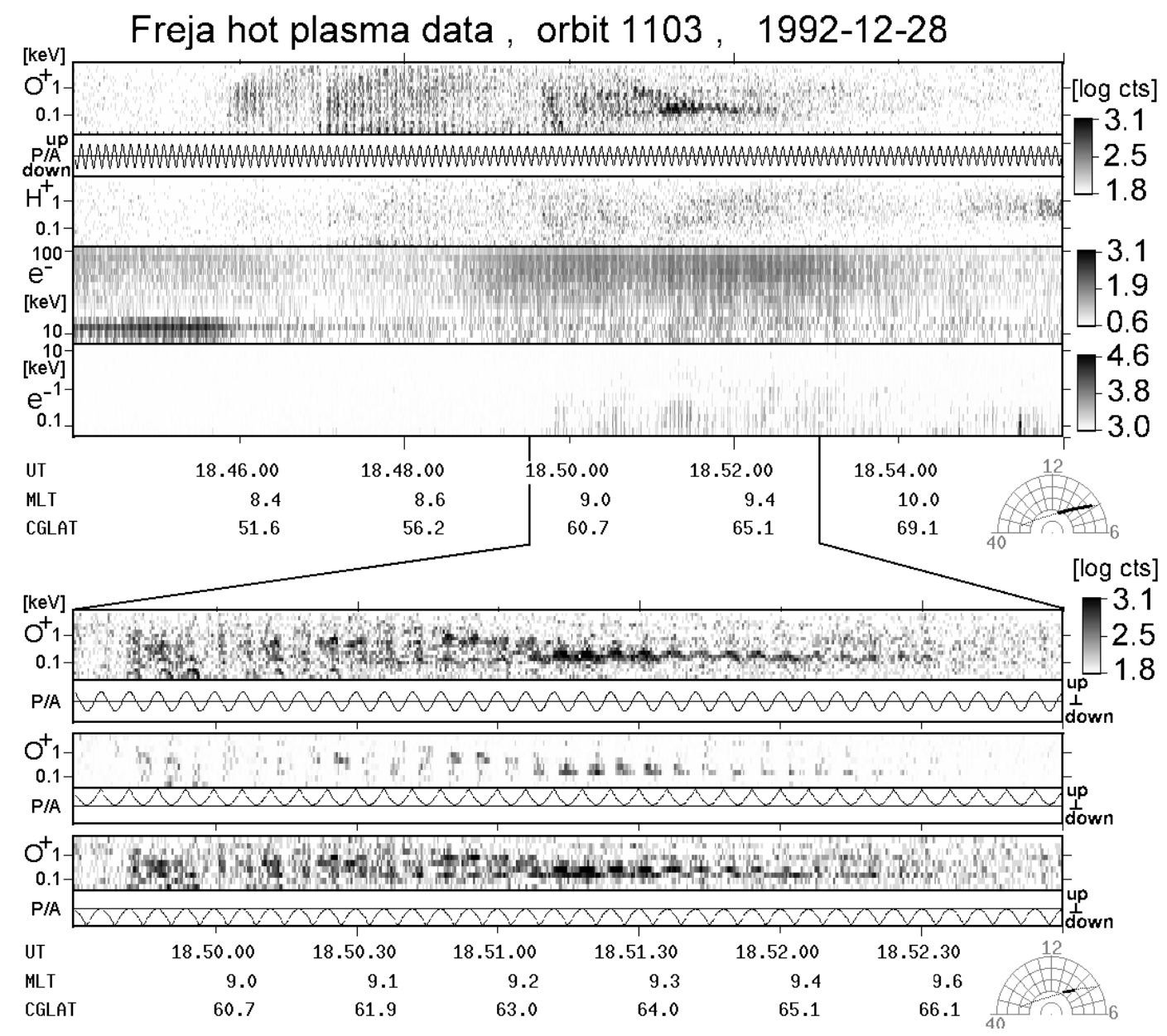

Fig. 6. Ion and electron spectrogram for Freja orbit 1103 (28 December 1992) in the same format as Fig. 1, except that we have three extra panels, one (third panel) showing 10-100 keV electrons (the same pitch angles for ions), and the other two showing heavy ions from sectors covering the upward direction (last second panel) and the downward direction (last panel) during a spin. $K_{p}=6-$ : this is one of the strongest magnetic storms during the Freja operation period.

are some possible explanations for this FAST-Freja discrepancy:

1. If the oxygen energy inside the cusp during magnetic storms is higher than the upper energy threshold of Freja, we see O+ only with FAST. This is possible if the cusp $\mathrm{O}+$ is of ring-current origin escaping through the dayside magnetopause into the magnetosheath. McFadden et al. (2003) showed one example with $10 \mathrm{keV} \mathrm{O+}$, one example with $1.5 \mathrm{keV} \mathrm{O}+$ and one example of several $\mathrm{keV} \mathrm{O+}$, but no statistics of the energy distribution.

2. If mirror reflection and charge-exchange loss between the Freja altitude $(1700 \mathrm{~km})$ and the FAST altitude is more important for $\mathrm{O}+$ than for $\mathrm{H}+$, most of $\mathrm{O}+$ disappears before it reaches the Freja altitude.

3. If thermalization between the FAST altitude and the Freja altitude is very strong, $\mathrm{H}+$ at the Freja altitude can be registered in a wide energy range, making the high fluxes of $\mathrm{H}+$ spill over the $\mathrm{O}+$ channel in Freja, while $\mathrm{H}+$ and $\mathrm{O}+$ are separated in energy at the FAST altitude.

4. $\mathrm{O}+$ can also contaminate the $\mathrm{H}+$ channel. If such an effect appears only in Freja, we cannot single out the $\mathrm{O}+$ signature from the widespread $\mathrm{H}+$ signature.

5. It could also be that strong $\mathrm{O}+$ injection occurs only for special magnetic storms.

At present we have no answer to the FAST-Freja discrepancy. To solve this, we need another low-altitude satellite $(<2000 \mathrm{~km})$ with a mass-resolving ion spectrometer with high energy $(\Delta \mathrm{E} / \mathrm{E}<10 \%)$ and pitch-angle $(<10 \mathrm{deg})$ resolution over the energy range of 0.1 to $40 \mathrm{keV} /$ charge and full pitch-angle range, and with spillover suppression (e.g. coincidence techniques).

\subsection{Multiple heavy ion dispersion event}

Figure 6 shows another unusual dayside event: multiple heavy ion dispersions on 28 December 1992 (orbit \#1103) 
during a major magnetic storm (see Fig. 4b for geomagnetic indices). The satellite traversed from the morning sector (8 MLT) to the evening sector (17 MLT) over the Northern Hemisphere. The energetic electron data (this was available during first few months of the mission) shows intensification of unstructured thermal $10^{2} \mathrm{keV}$ electrons at 18:4918:54 UT, indicating that this is probably the outer radiation belt. In the lower panels of the figure where the blow-up data between 18:49:30-18:53:00 UT are presented for the oxygen channel, we also included data from sectors that cover the field-aligned upward direction (last second panel) and the field-aligned downward direction (last panel).

One can recognize two outstanding features in the heavy ion channel: one is the wedge-like dispersed structure of trapped oxygen at around 18:47 UT (52-58 CGLat) far equatorward of the auroral region, and the other is the overlapping and diverging energy-time dispersions (weak dispersion earlier and strong dispersion later) at around 18:50 UT (6165 CGLat) which is probably inside the radiation belt. The former has been reported in Yamauchi et al. (1996a, 2005), and this type of energy-latitude dispersion can be understood as a signature of drifting ions several hours after the substorm injections (Ebihara et al., 2001).

The latter event (18:50 UT) is the one we discuss here. Between 18:50:10 UT and 18:52:30 UT, we see multiple injections (downward direction is more intense than upward direction) with different dispersion strength. Meanwhile, the proton channel showed very low counts during this interval, indicating that this is exclusively a heavy ion $(\mathrm{O}+$ or $\mathrm{N}+)$ phenomenon.

There are three dispersions visible at 18:50:10 UT (from $0.56 \mathrm{keV}$ to $0.13 \mathrm{keV}$ in 2 spins or $12 \mathrm{~s}$ ), 18:50:25 UT (from $1.0 \mathrm{keV}$ to $0.24 \mathrm{keV}$ in 4 spins or $24 \mathrm{~s}$ ), and 18:50:40 UT (from $2.4 \mathrm{keV}$ to $0.32 \mathrm{keV}$ in $13-14$ spins or $75 \mathrm{~s}$ ). From these dispersion curves, one can obtain the time-of-flight (for fixed pitch angles) as $10 \mathrm{~s}, 25 \mathrm{~s}$, and $45 \mathrm{~s}$, indicating that the start times of all these dispersions fall within 18:49:5518:50:00 UT, i.e. a simultaneous starting time for all injections. Such a converging multiple dispersion was never observed again in more than 2 years of Freja operation. The time-of-flight distance is estimated to be less than $1000 \mathrm{~km}$.

The dispersed ions are detected rather in the oblique direction than in the downgoing direction (perpendicular component is larger than parallel component), and furthermore they are detected mainly during one of two half-spin periods. In other words, these ions are highly non-gyrotropic. This is quite different from the ordinary injection events. Since the oxygen gyrofrequency at the Freja altitude is of the order of one hundred $\mathrm{Hz}$, the time-of-flight calculated above is long enough for phase-mixing through instabilities to make the injection gyrotropic. This is not the case with the observed injection event. Then one might question if it is really an injection event. Since perpendicular heating is commonly observed by Freja (Eliasson et al., 1994), this event could also be one special form of heating signature regardless of its low latitude (61 CGLat) and downgoing flux. Actually, one can recognize intensive wave activity during this event.
Electromagnetic wave activities and ion heatings are generally strongly related (Andre et al., 1998).

However, interpreting this event as a wave signature has difficulties. In addition to its downgoing flux, the nongyrotropic distribution can hardly be explained by wave theory. The non-gyrotropic distribution with downgoing flux instead suggests that: 1) perpendicular convection (of the entire plasma) is extremely large (comparable to the perpendicular $\mathrm{O}+$ velocity, i.e. nearly $100 \mathrm{~km} / \mathrm{s}$ ), 2) the source of the injection is very close within a distance corresponding to only several to several tens gyro period (no more than $1 \mathrm{~s}$ for $\mathrm{O}+$, i.e. less than $100 \mathrm{~km}$ ), or 3 ) the anisotropy of the source distribution was maintained during its travel for nearly a minute. The source pitch-angle distribution must be very anisotropic (beam-like) in all cases.

The first possibility (fast convection) is very unlikely because we did not detect any signature of such a fast convection in the field data or proton data. However, we have no means of eliminating the other possibilities. Let us point out that the difference in the dispersion curve means that the mean pitch angles of the beam-like sources are quite different for the three injections, although all three dispersions are composed of particles with large pitch angles (45 90 deg). In other words, the source distribution could have three peaks at different pitch angles if three injections come from the same region, i.e. for the third possibility. In this case, at least the second and the third dispersion must be dominated by the perpendicular component, which is consistent with the observation.

Thus, the pitch-angle anisotoropy is consistent with injection from a single source at a given time, although it is also quite possible that three injections have simultaneous but separated sources very close to the spacecraft $(<100 \mathrm{~km}$ distance). If this event originates from a single source, a sudden localized energization of dense oxygen should have taken place within $1000 \mathrm{~km}$ distance from the spacecraft. In either case the short source distance makes it difficult for other spacecraft (e.g. Viking and FAST) to detect this type of phenomenon, although they carry mass-resolving ion spectrometers. It is also difficult to observe the phenomenon using the ground magnetometers if it really is localized energization because the nearest station, Victoria (49 GGLat, 137 Long) is more than $1000 \mathrm{~km}$ away from the footpoint. The ground magnetometer indeed did not show any specific intensification.

\section{Summary and conclusions}

We have surveyed almost the entire hot plasma data (1992.10-1994.10) from Freja for heavy ion injection events. Although the satellite inclination $\left(63^{\circ}\right)$ reduces the chance of covering the dayside polar cap boundary, the nightside preference of heavy ion injections is well demonstrated by the Freja data. We have also reported unusual heavy ion events in the dayside during major magnetic storms. 
1. On 21 February 1994, a mono-energetic heavy ion injection (decreasing energy-time dispersion) was observed at 13 MLT followed by proton injections with the same velocity (mass ratio is 15-20). These heavy ions are most likely oxygen $(\mathrm{O}+)$. The time-of-flight distance is $1 \mathrm{Re}$ and the velocity-filtered source distance is more than $10 \mathrm{Re}$. The southern dayside ionosphere is the most probable source because the strongly dawnward IMF during this period could have moved the southern cusp near the conjugate point of the injection region. The exact mechanism that provides the energy and mass filter is unknown.

2. On 28 December 1992, an overlapping heavy ion injection in an oblique non-gyrotropic direction was observed in the middle of the outer radiation belt. The diverging dispersion and the pitch-angle distribution indicate a sudden localized anisotropic energization of dense oxygen near $(<1000 \mathrm{~km})$ and above the spacecraft, although we cannot tell if different injections come from a single source or from several sources with a simultaneous start time. In the case of the single source, the source distribution must be very anisotropic with at least 3 sharp peaks at different pitch angles. The generation mechanism is completely unknown.

Acknowledgements. The Freja project is supported by the Swedish National Space Board and the Deutsche Agentur für Raumfahrtangelegenheiten. The geomagnetic indices are provided by WDC-C2 for geomagnetism at Kyoto University. M. Yamauchi thanks programs for disabled people in Sweden which have made it possible for him to work.

Topical Editor T. Pulkkinen thanks D. Winningham and another referee for their help in evaluating this paper.

\section{References}

Andre, M., Norqvist, P., Andersson, L., Eliasson, L., Eriksson, A. I., Blomberg, L., Erlandson, R. E., and Waldemark, J.: Ion energization mechanisms at $1700 \mathrm{~km}$ in the auroral region, J. Geophys. Res., 103, 4199-4222, 1998.

Blanc, M., Horwitz, J. L., Blake, J. B., Daglis, I., Lemaire, J. F., Moldwin, M. B., Orrsini, S., Thorne, R. M., and Wolf, R. A.: Source and loss processes in the inner magnetosphere, Chapter 4 in: Magnetospheric Plasma Sources and Losses, edited by Hultqvist, B., Øieroset, M., Paschmann, G., and Treumann, R., Kluwer Academic Pub., 137-206, 1999.

Boehm, M., Paschmann, G., Clemmons, J., Höfner, H., Fremzel, R., Ertl, M., Haerendel, G., Hill, P., Lauche, H., Eliasson, L., and Lundin, R.: The TESP electron spectrometer and correlator (F7) on Freja, Space Sci. Rev., 70, 509-540, 1994.

Bosqued, J., Sauvaud, J. A., Delcourt, D., and Kovrazhkin, R. A.: Precipitation of suprathermal ionospheric ions accelerated in the conjugate hemisphere, J. Geophys. Res., 91, 7006-7018, 1986.

Carlson, C., Pfaff, R., and Watzin, J.: The Fast Auroral SnapshoT (FAST) mission, Geophys. Res. Lett., 25, 2013-2016, 1998.

Chappell, C. R., Olsen, R. C., Green, J. L., Johnson, J. F. E., and Waite, Jr., J. H.: The Discovery of nitrogen ions in the Earth's magnetosphere, Geophys. Res. Lett., 9, 937-940, 1982.
Ebihara, Y., Yamauchi, M., Nilsson, H., Lundin, R., and Ejiri, M.: Wedge-like dispersion of sub-keV ions in the dayside magnetosphere: Particle simulation and Viking observation, J. Geophys. Res., 106, 29571-29584, 2001.

Eliasson, L., Norberg, O., Lundin, R., Lundin, K., Olsen, S., Borg, H., Andre, M., Koskinen, H., Riihela, P., Boehm, M., and Whalen, B.: The Freja hot plasma experiment - instrument and first results, Space Sci. Rev., 70, 563-576, 1994.

Frahm, R. A., Reiff, P. H., Winningham, J. D., and Burch, J. L.: Banded ion morphology: Main and recovery storm phases, in: Ion Acceleration in the Magnetosphere and Ionosphere, ed. by Chang, T. S., 98-107, AGU, Washington, D.C., 1986.

Hamilton, D. C., Gloeckler, G., Ipavich, F. M., Studemann, W., Wilken, B., and Kremser, G.: Ring current development during the great geomagnetic storm of February 1986, J. Geophys. Res., 93, 14343-14355, 1988.

Hirahara, M., Yamazaki, A., Seki, K., Mukai, T., Sagawa, E., Kaya, N., and Hayakawa, H.: Characteristics of downward flowing ion energy dispersions observed in the low-altitude central plasma sheet by Akebono and DMSP, J. Geophys. Res., 102, 4821-4839, 1997.

Hultqvist, B.: Downward ion acceleration at auroral latitudes: cause of parallel electric field, Ann. Geophys., 20, 1117-1136, 2002,

SRef-ID: 1432-0576/ag/2002-20-1117.

Kletzing, C. A. and Torbert, R. B.: Electron time dispersion, J. Geophys. Res., 99, 2159-2172, 1994.

Lundin, R., Eliasson, L., Hultqwvist, B., and Stasiewicz, K.: Plasma energization on auroral field lines as observed by the Viking spacecraft, Geophys. Res. Lett., 14, 443-446, 1987.

Mauk, B. H.: Quntitative modeling of the "convection surge" mechanism of ion acceleration, J. Geophys. Res., 91, 13 423-13431, 1986.

McFadden, J. P., Carlson, C. W., Strangeway, R., and Moebius, E. Observations of downgoing velocity dispersed $\mathrm{O}+$ and $\mathrm{H}+$ in the cusp during maggnetic storms, Geophys. Res. Lett., 30, 19471951, doi: 10.1029/2003GL017783, 2003.

Moore, T. E., Lundin, R., Alcayde, D., Andre, M., Ganguli, S. B., Temerin M., and Yau, A.: Source processes in the high-latitute ionosphere, Chapter 2 in 'Magnetospheric Plasma Sources and Losses", edited by Hultqvist, B., Øieroset, M., Paschmann, G., and Treumann, R., Kluwer Academic Pub., 7-84, 1999.

Norberg, O., Yamauchi, M., Eliasson, L., and Lundin, R.: Freja observations of multiple injection events in the cusp, Geophys. Res. Lett., 21, 1919-1922, 1994.

Norqvist, P., Andre, M., and Tyrland, M.: A statistical study of ion energization mechanisms in the auroral region, J. Geophys. Res., 103, 23 459-23 474, 1998.

Quinn, J. M. and McIlwain, C. E.: Bouncing ion clusters in the Earth's magnetosphere, J. Geophys. Res., 84, 7365-7370, 1979.

Shelley, E. G., Johnson, R. G., and Sharp, R. D.: Satellite observations of energetic heavy ions during a geomagnetic storm, J. Geophys. Res., 77, 6104-6110, 1972.

Winningham, D. J., Yasuhara, F., Akasofu, S.-I., and Heikkila, W. J.: The latitudinal morphology of $10 \mathrm{eV}$ to $10 \mathrm{keV}$ electron fluxes during quiet and disturbed times in the 21-03 MLT sector, J. Geophys. Res., 80, 3148-3171, 1975.

Winningham, D. J., Burch, J. L., and Frahm, R. A.: Bands of ions and angular V's: A conjugate manifestation of ionospheric ion acceleration, J. Geophys. Res., 89, 1749-1754, 1984.

Woch, J. and Lundin, R.: The low-latitude boundary layer at midaltitudes: Identification based on Viking hot plasma data, Geophys. Res. Lett., 20, 979-982, 1993. 
Yamauchi, M., Woch, J., Lundin, R., Shapshak, M., and Elphinstone, R.: A new type of ion injection event observed by Viking, Geophys. Res. Lett., 20, 795-798, 1993.

Yamauchi, M., Lundin, R., Eliasson, L., and Norberg, O.: Mesoscale structures of radiation belt/ring current detected by lowenergy ions, Adv. Space Res., 17, 171-174, 1996a.

Yamauchi, M., Nilsson, H., Eliasson, L., Norberg, O., Boehm, M., Clemmons, J. H., Lepping, R. P., Blomberg, L., Ohtani, S.-I., Yamamoto, T., Mukai, T., Terasawa, T., and Kokubun, S.: Dynamic response of the cusp morphology to the solar wind: A case study during passage of the solar wind plasma cloud on 21 February 1994, J. Geophys. Res., 101, 24 675-24 687, 1996 b.
Yamauchi, M., Lundin, R., Eliasson, L., Winningham, D., Reme, H., Vallat, C., Dandouras, I., and Cluster-CIS team: Structures of sub-keV ions inside the ring current region, in: Physics and Modeling of the Inner Magnetosphere, edited by Pulkkinen, T. I., Tsyganenko, N. A., and Friedel, R., AGU Monoraph, in press, 2005.

Yau, A. W., Whalen, B. A., Goodenough, C., Sagawa, E., and Mukai, T.: EXOS-D (Akebono) observations of molecular NO+ and $\mathrm{N} 2+$ upflowing ions in the high- altitude auroral ionosphere, J. Geophys. Res., 98, 11 205-11224, 1993. 